Vol. 3, No. 1 | January - June 2020

\title{
Illicium verum as a green source for the synthesis of silver nanoparticles and investigation of antidiatom activity against Paeodactylum Tricornutum
}

\author{
Muhammad Nazim Lakhan ${ }^{1}$, Rongrong Chen ${ }^{1}$, Altaf Hussain Shar ${ }^{1}$, Kishore \\ Chand $^{1}$, Muhammad Basit Chandio ${ }^{2}$, Ahmer Hussain Shah ${ }^{3}$, Mukhtiar \\ Ahmed ${ }^{1}$, Rizwan Ahmed ${ }^{4}$, Jun Wang ${ }^{1}$
}

\begin{abstract}
:
Biological fouling has caused a lot of concern in marine industries due to the attachment of microorganisms including bacteria, algae, and diatoms on a marine surface to create a biofilm. Biofouling causes negative impacts on the marine industry such as an increase in weights of hulls, low speed, and high fuel consumption. In the recent past, nanoparticles have attracted a lot of attention in the fields of material science, chemistry, and biology owing to their rare biological properties. Silver nanoparticles (AgNPs) have been long known for its strong toxicity against a wide range of microorganisms. Herein, we synthesized the AgNPs via a green synthesis approach known for its benefits such as one-pot, inexpensive, and eco-friendly; by using Illicium verum (IV) extract as a demoting and sustaining agent. Further, characterization tests of obtained AgNPsIV were investigated including Ultraviolet-visible spectroscopy (UV-Vis), Fourier-transform infrared spectroscopy (FTIR), X-Ray Diffraction (XRD), Scanning electron microscopy (SEM), Transmission electron microscopy (TEM) and Atomic force microscopy (AFM). The UV-Vis result confirmed the AgNPs-IV formation with its surface Plasmon resonance peak. FTIR was tested to investigate the bio-functional groups liable for the AgNPs-IV synthesis. XRD peaks also meet with the standard of AgNPs (JCPDS: 41-1402). SEM, TEM, and AFM analysis of AgNPsIV showed the hexagonal structure with $14.56 \mathrm{~nm}$ mean size. The cell growth of diatom on $5^{\text {th }}$ day with blank sample suspension was 2.308 (Cell $10^{5}$ number $/ \mathrm{mL}$ ), while for AgNPs-IV sample suspension was 0.19 (Cell $10^{5}$ number $/ \mathrm{mL}$ ). The green synthesized AgNPs-IV showed excellent antidiatom activity against Paeodactylum Tricornutum (P. Tricornutum) marine diatom.
\end{abstract}

Keywords: Nanotechnology; silver nanoparticles; illicium verum; synthesis; characterization.

${ }^{1}$ Key Laboratory of Superlight Material and Surface Technology, Ministry of Education, Harbin Engineering University, People's Republic of China

${ }^{2}$ College of Mechanical and Electrical Engineering, Harbin Engineering University, People's Republic of China

${ }^{3}$ Department of Textile Engineering, Balochistan University of Information Technology, Engineering and Management Sciences, Quetta, Pakistan

${ }^{4}$ School of Chemical Engineering, Dalian University of Technology, People's Republic of China Corresponding Author; nazimlakhan@gmail.com SJET | ISSN: 2616-7069 | Vol. 3 | No. 1 | @ 2020 Sukkur IBA University 


\section{Introduction}

Biological fouling is the accumulation of algae, plants, and microorganisms on marine surfaces immersed in seawater. Antifouling is the ability of specifically designed materials to prevent biofouling by various numbers of microorganisms such as algae, fungi, and diatoms. Development of eco-friendly and facile green synthesis of metallic nanoparticles (having size $<100$ $\mathrm{nm}$ ) has attained huge attention in recent days due to their ample areas of biological applications including antibacterial, diagnostics, and drug delivery systems [13]. Metallic nanoparticles possess chemical and physical properties including a surface area to volume ratio and exhibit strong antibacterial property [4-5]. Among the others, silver has received extreme importance due to its strong toxicity against microorganisms. Currently, silver nanoparticles (AgNPs) are considered as rich particles with their applications in various areas including antimicrobial and antioxidant properties [6-8]. There are several approaches to AgNPs synthesis such as thermal decomposition, microwave irradiation, electrochemical, and biological. Among them, the biological method has many benefits such as simple, less cost, and eco-friendly for large scale applications that do not use toxic chemicals, high temperature, and pressure [9]. Interestingly, AgNPs can be synthesized by using enzymes, proteins, microorganisms, and plant materials. Plant extract synthesis is considered as an efficient approach than chemical and microbial synthesis because plant works as demoting and sustaining agent in the preparation of AgNPs and the particles formed are more stable with different shapes and sizes. Herein, we have used northeast Vietnam and southwest China evergreen tree plant fruit Illicium verum, also known as "Chinese star anise" as a demoting and sustaining agent. Star anise has been used in China for flavoring and medicine purposes for over three thousand years. It has been widely used in the medicines system and contains the principal constituent Anethole which possesses antiviral, antibacterial, antifungal, and antioxidant activity.
In this study, the AgNPs-IV synthesized via a green approach using an extract of IV as a demoting and sustaining agent. The asprepared AgNPs-IV were characterized by different techniques such as UV-Vis, FTIR, XRD, SEM-EDX, TEM, and AFM. Furthermore, this is the first report of AgNPs-IV and its antidiatom activity against marine diatom $P$. tricornutum in the marine environment.

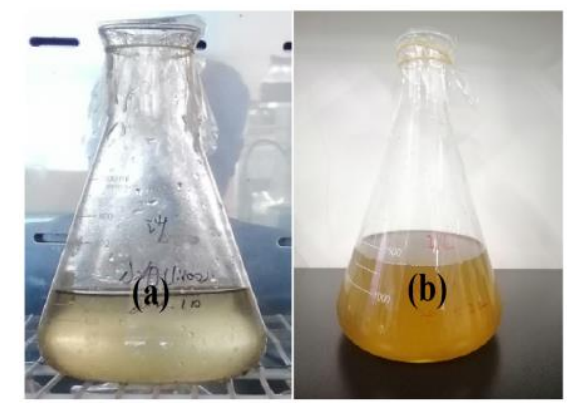

Fig. 1. (a) Blank and (b) P. tricornutum diatom growth solution

\section{Materials and Method}

\subsection{Materials}

The fresh plant Illicium verum (IV) fruit was obtained from the local market of Harbin, China, and grinded in powder form. The silver nitrate $\left(\mathrm{AgNO}_{3}\right)$ and ethanol $\left(\mathrm{C}_{2} \mathrm{H}_{6} \mathrm{O}\right)$ were received from Tianjing Chemical Co., China. Distilled water was used to wash the equipment and later dried in the oven. Materials used in the synthesis process were of analytical grade.

\subsection{Preparation of Plant extract}

$0.5 \mathrm{~g}$ of IV powder was weighted and dissolved with $400 \mathrm{ml}$ of Milli-Q water and then the solution was boiled at $60^{\circ} \mathrm{C}$ for 20 min to obtain its aqueous extract. After cooling at room temperature the IV extract was filtered via Whatman No.1 and stored in the refrigerator at $4{ }^{\circ} \mathrm{C}$ for further experimental study.

\subsection{Biosynthesis of AgNPs-IV with Illicium verum extract}

For the green synthesis of AgNPs-IV, the solution of 1 molarity was obtained by dissolving weighed amount of $\mathrm{AgNO}_{3}$ in $400 \mathrm{ml}$ of distilled (DI) water under 
Lakhan M.N (et al.), Illicium verum as a green source for synthesis of silver nanoparticles and investigation of their antidiatom activity against Paeodactylum Tricornutum diatom (pp. $23-30)$

sonication. Then the as-prepared $80 \mathrm{ml}$ of extract IV was added in $\mathrm{AgNO}_{3}$ aqueous solution drop by drop for reduction of $\mathrm{Ag}^{+}$ into $\mathrm{Ag}^{0}$ under the continuous magnetic stirring. The mixed solution was placed in a dark environment for $24 \mathrm{hrs}$ to minimize the photoactivation of $\mathrm{AgNO}_{3}$. After the successful synthesis of the AgNPs-IV solution, AgNPs-IV was centrifuged and washed two times for $15 \mathrm{~min}$ at $8000 \mathrm{rpm}$ with DI water. Later, the as-obtained AgNPs-IV was placed in a vacuum oven overnight at $60^{\circ} \mathrm{C}$ and collected for further characterization and antidiatom activity.

\subsection{Characterization}

The absorption spectrum of the synthesized AgNPs-IV was investigated by UV-Vis spectrophotometer TU-1901 dual-beam in the range of 200-800 nm. FTIR analysis was carried out using a Perkin Elmer spectrum 100 FTIR spectrometer in the wave number range of $4000-500 \mathrm{~cm}^{-1}$ to identify the chemical constituents liable for the successful preparation of AgNPs-IV. XRD pattern was examined to check the nature of the as-obtained samples by using Rigaku TTR at $40 \mathrm{kV}$ and $150 \mathrm{~mA}$ within $2 \theta$ area between $10-90^{\circ}$ with intensity $\mathrm{Cu}$ $\mathrm{Ka}$ radiation $(\lambda=0.15406 \mathrm{~nm})$. The morphology of as-obtained AgNPs-IV was viewed by the SEM instrument (JOEL, JSM-6480A) operated at a $20 \mathrm{KV}$ of the driving voltage. Element and composition analysis of the powdered AgNPs-IV was conducted using a JEOL's EDX detector attached to the SEM machine. The size of as-obtained AgNPs-IV was observed by TEM using an FEI TECHNI G2 instrument. The topography of AgNPs-IV was investigated by AFM using a Keysight equipment Model 5500. The average mean size of nanoparticles was measured by Nano Measurer Software.

\subsection{Antidiatom Analysis}

The algae $P$. Tricornutum were examined for the anti-diatom test to check the antifouling performance of AgNPs-IV. The algal stock solution of $P$. Tricornutum was received from Xiamen UniversityCCMA and it was growth in F/2 medium with a 12/12 light, dark cycle of fluorescent illumination at 2000 lux and growth sample solutions were shaken daily twice a day. Blank sample and green synthesized AgNPs-IV (5mL) solution was poured in the algal solution at the bottom of the aquarium with at least $1 \times 10^{5}$ algal cells per $\mathrm{mL}$ and blank substrate was placed in both beakers to confirm the morphology of surface after 5 days' immersion, later the beakers were placed in an incubator [10]. The diatom settling assay of blank and AgNPs-IV samples was recorded on an Optical microscope.

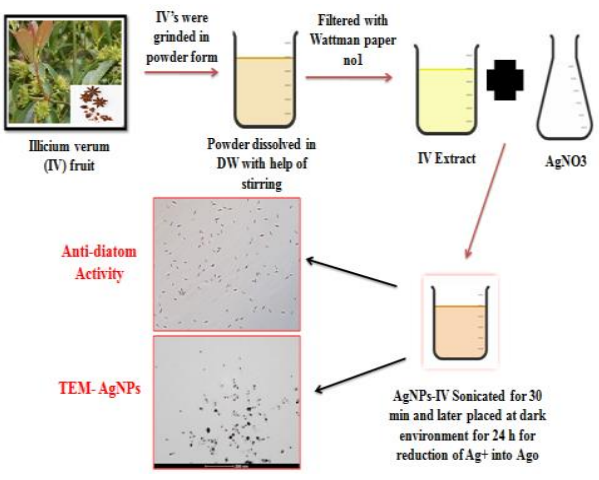

Fig. 2. Schematic route of AgNPs formation using plant extract of illicium verum (IV).

\section{Results and discussion}

The successful synthesis of AgNPs-IV was observed on UV-Vis spectrum through the color formation. The color of the $\mathrm{AgNO}_{3} /$ Illicium verum extract mixture turned from light to dark brown indicates the successful formation of AgNPs-IV. The dark brown color appeared due to the excitation of surface plasmon resonance (SPR) effect. Further, the formation of the AgNPs-IV was observed by the SPR absorption band at $425 \mathrm{~nm}$ in the UV-Vis region as depicted in Fig. 3 [11].

Further, AgNPs-IV were characterized by FTIR spectra to analyze the functional groups liable for demoting and sustaining of Ag nanoparticles as depicted in Fig. 4. The FTIR spectrum shows different main peaks at 581, 1118, 1634, 2357, and $3459 \mathrm{~cm}^{-1}$. The peak appeared in the spectrum at 1634 $\mathrm{cm}^{-1}$ clearly shows the stretching vibration of $\mathrm{C}=\mathrm{O}$ and indicating the formation of 
AgNPs-IV and capped with the biofunctional groups [12]. The peak at 2357 $\mathrm{cm}^{-1}$ and $1157 \mathrm{~cm}^{-1}$ represents the $\mathrm{O}-\mathrm{H}$ and C-O stretching. The peak at $581 \mathrm{~cm}^{-1}$ indicates the existence of alkaloids. The peak at $3459 \mathrm{~cm}^{-1}$ was assigned to $\mathrm{NH}$ stretching vibration of amide group and aromatic rings [13].

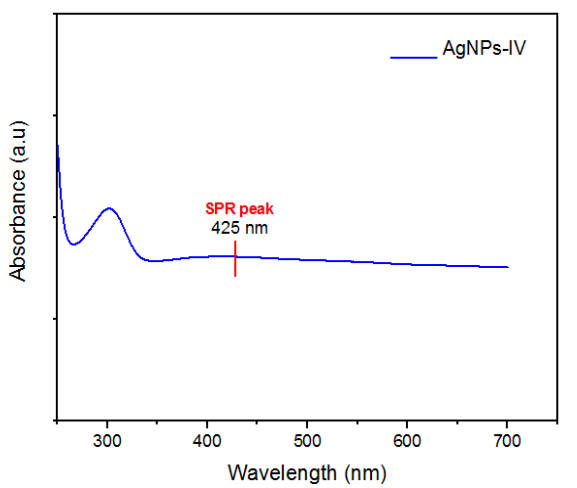

Fig. 3. UV-Vis Spectrum of AgNPs-IV.

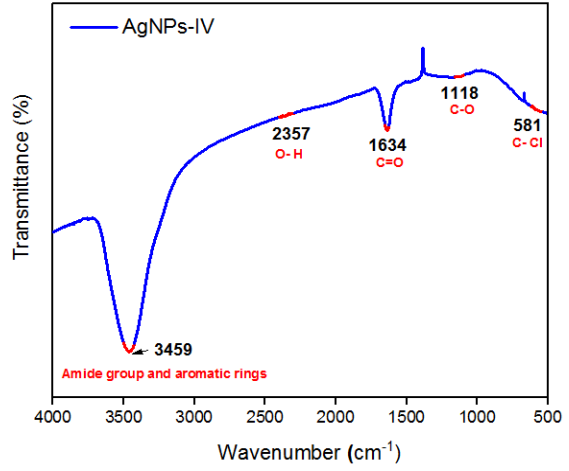

Fig. 4. FTIR Spectrum of AgNPs-IV.

The XRD pattern of AgNPs-IV is displayed in Fig. 5. Several different diffraction peaks were observed at $2 \theta$ of approximately $35.89^{\circ}, 37.04^{\circ}, 51.68^{\circ}, 55.07^{\circ}, 59.13^{\circ}$, $67.52^{\circ}$ and $76.08^{\circ}$ corresponding to reflections from the (004), (101), (104), (006), (105), (112) and (008) crystallographic planes of AgNPs-IV, respectively, and confirming the hexagonal structure of silver (JCPDS, No. 41-1402) $[14,15]$.

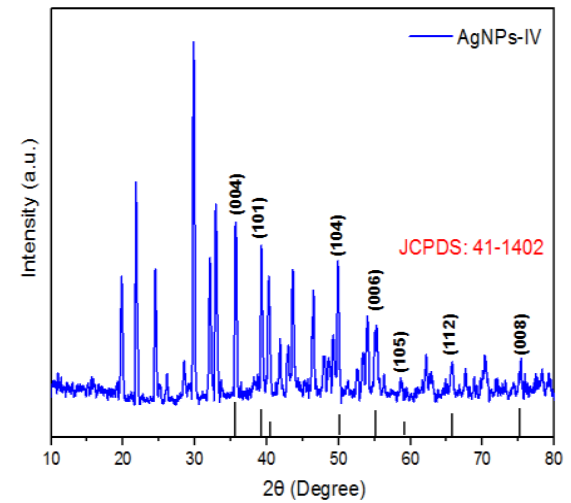

Fig. 5. XRD Pattern of AgNPs-IV.

The morphology of the as-obtained AgNPsIV was observed by SEM as shown in Fig. 6(a). It was seen that the larger particles are also formed with smaller particles which indicates the aggregation in $\mathrm{Ag}$ particles and the evaporation of solvent while sampling preparation [16]. The EDX spectrum of AgNPs-IV showed strong signal at $3 \mathrm{keV}$ [17]. In Fig. 6(b), the peak for $\mathrm{Cl}$ was observed due to the presence of plant extract, while the peak of $\mathrm{Cu}$ was due to the carbon grid that was used during sample preparation.

The morphology and size of the AgNPs-IV were examined by TEM and AFM. The obtained TEM result illustrated that silver nanoparticles are readily oxidized due to the functional compounds present in the plant extract and successfully formed with hexagonal in shape as depicted in Fig. 7(a) [18-19]. In Fig. 7(b), the measured average mean size of nanoparticles was $14.56 \mathrm{~nm}$ obtained through Nano Measurer software using the TEM image. The dark shades on the surface of nanoparticles revealed the presence of plant extract. The AFM image in Fig. 7(c) showed that the particles are formed with little aggregation and marked lines showing the line size of particles at different positions. The particle size curve was obtained through NanoScope Analysis software as shown in Fig. 7(d). The AFM analysis has similarities with the TEM and SEM results. 
Lakhan M.N (et al.), Illicium verum as a green source for synthesis of silver nanoparticles and investigation of their antidiatom activity against Paeodactylum Tricornutum diatom
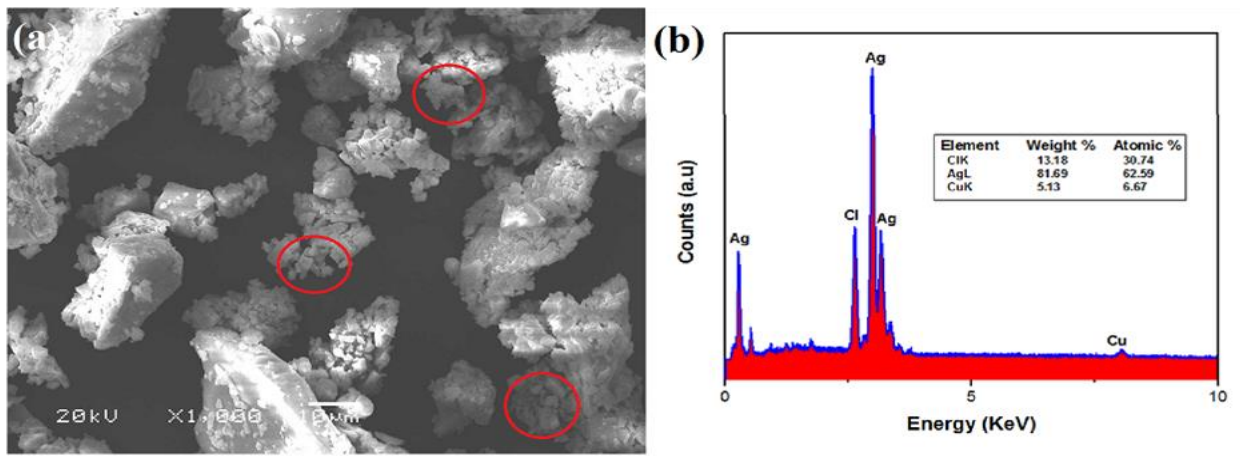

Fig 6. Scanning electron microscopy image of AgNPs-IV (a), EDX Spectrum of AgNPs-IV (b)
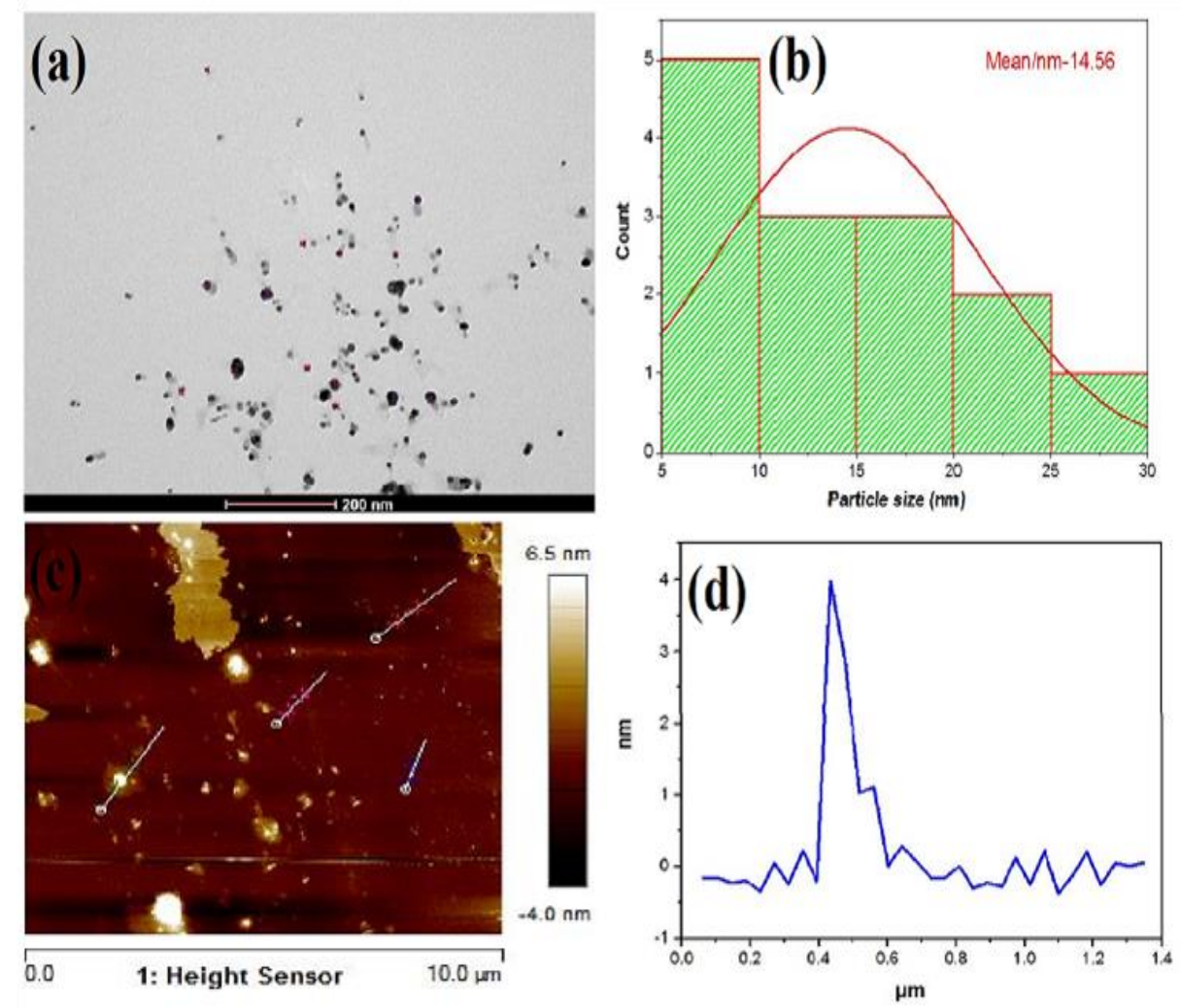

Fig. 7. Transmission electron microscopy image at $200 \mathrm{~nm}$ (a), particle size distribution through TEM image (b), atomic force microscopy image (c), and Particle size obtained through AFM image (d) of AgNPs-I

Diatoms are microscopic algae that grow on manmade surfaces present in the seawater. Biofouling develops due to the attachment of diatoms, bacteria, and other microorganisms on the surfaces in seawater. The anti-settling test of diatom was analyzed to check the antifouling performance of the blank sample and AgNPs-IV. Therefore, herein, the $P$. tricornutum diatom was tested to check the biofouling attachemnt. The inhibition growth effect of AgNPs-IV was observed in the $P$. tricornutum suspension [10]. From the anti-settling analysis, it was seen that 
Lakhan M.N (et al.), Illicium verum as a green source for synthesis of silver nanoparticles and investigation of their antidiatom activity against Paeodactylum Tricornutum diatom (pp. $23-30)$

the more algae cells were found on a blank sample, while AgNPs IV surface was attached with the no algae cells after 5 days' immersion as shown in Fig. 8(a-d). The diatom attachment was checked in suspension sample by an optical microscope attached with the cell count chamber haemocytometer. Fig. 9 showed the cell number of diatoms in the $P$. tricornutum suspension on day 1 and after cultivation for 5 days. The absorbance of diatoms was less for AgNPs-IV sample, which was observed on Uv-Vis spectroscopy at $600 \mathrm{~nm}$ in algae solution, while for blank samples; its absorbance was almost the same after five days' period.

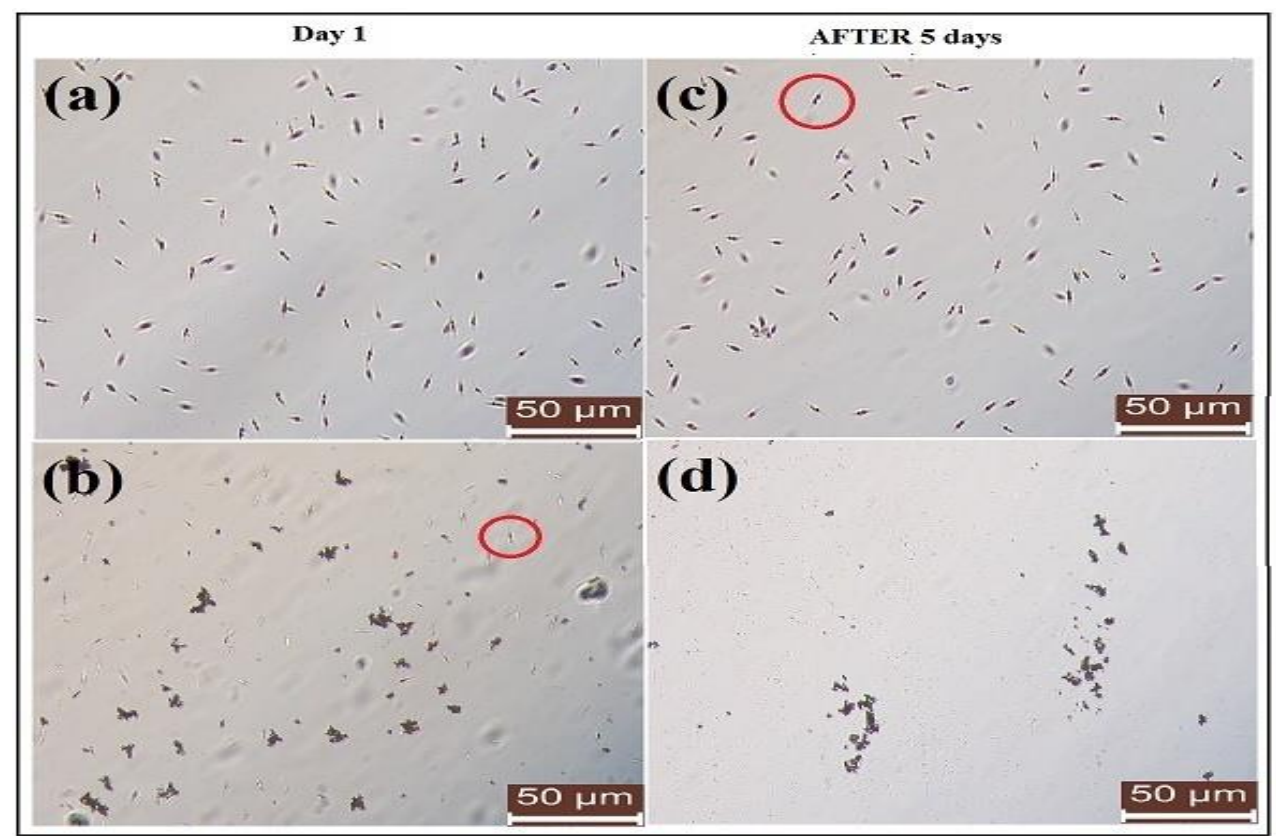

Fig. 8. Antidiatom activity of blank sample on (a) day 1, (c) day 5, and AgNPs-IV 5ml sample on (b) day 1 and (d) day 5 .

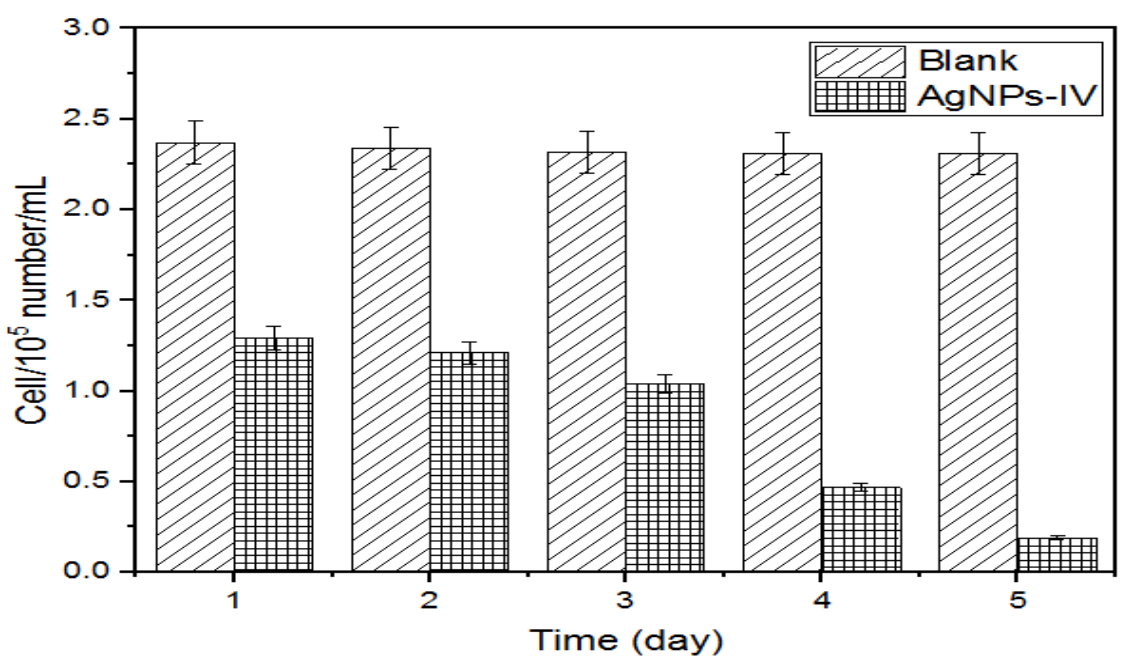

Fig. 9. The cell number of diatoms (P. tricornutum) in the solutions with blank and AgNPs-IV samples. Each point is the average of three readings 
Lakhan M.N (et al.), Illicium verum as a green source for synthesis of silver nanoparticles and investigation of their antidiatom activity against Paeodactylum Tricornutum diatom (рp. $23-30)$

\section{Conclusion}

In this article, we presented a green route for the synthesis of the silver nanoparticles from Illicium verum extract. The amount of plant extract plays a vital role in the preparation of well-dispersed and small size nanoparticles and also acts as a reducing and stabilizing agent. The method employed here is one-pot, simple, inexpensive, and eco-friendly. The results confirm the uniform formation of small size nanoparticles with a hexagonal shape. The green synthesized AgNPs-IV showed excellent antidiatom activity against Paeodactylum Tricornutum diatom. In this regard, we can affirm that the AgNPs prepared by the green approach could be a potential tool in the near future to combat biofouling on manmade surfaces in seawater.

\section{ACKNOWLEDGMENT}

The author's greatly appreciated the financial support by the Fundamental Research Funds of the Central University (3072019CF1008), National Natural Science Foundation of China (NSFC 51603053), the Application Technology Research and Development Projects of Harbin (2015RAQXJ038) and Defense Industrial Technology Development Program (JCKY2018604C011).

\section{References}

[1].E. Pavitra, , B. Dariya, G. Srivani, S.M Kang, A. Alam, P.R Sudhir, M. A Kamald, G. S.R Raju, Y.K Han, B. V K. S Lakkakula, G. P Nagarajui, Y. S. Huh, Engineered nanoparticles for imaging and drug delivery in colorectal cancer, Seminars in cancer Biology, 2019, pp. 1-14.

[2].S. Ahmed, M. Ahmad, B. L. Swami, S. Ikram, A review on plants extract mediated synthesis of silver nanoparticles for antimicrobial applications: A green expertis, J Adv Res, Vol. 7, 2016, pp. 17-28.

[3].H. Chandra, P. Kumari, E. Bontempi, S. Yadav, Medicinal plants: Treasure trove for green synthesis of metallic nanoparticles and their biomedical applications, Biocatalysis and Agricultural Biotechnology, 2020, pp. 147.

[4].R. Dadi, R. Azouani, M. Traore, C. Mielcarek, A. Kanaev, Antibacterial activity of $\mathrm{ZnO}$ and $\mathrm{CuO}$ nanoparticles against gram positive and gram negative strains, Material Science \& Engineering C, Vol. 104, 2019, pp. 1-9.

[5].S. Kheiri, X. Liu, M. Thompson, Nanoparticles at biointerfaces: Antibacterial activity and nanotoxicology, Colloids and Surfaces B: Biointerfaces, 2019, pp. 1-43.

[6].A. Chatterjee, S. Khatua, K. Acharya , J. Sarkar, A green approach for the synthesis of antimicrobial bio- surfactant silver nanoparticles by using a fern, Digest Journal of Nanomaterials and Biostructures, Vol. 14, 2019, pp. 479-490.

[7].M. C. Biswas, B. J. Tiimob, W. Abdela, S. Jeelani, V. K. Rangari, Nano silica-carbonsilver ternary hybrid induced antimicrobial composite films for food packaging application, Food Packgaing and Shelf Life, Vol. 19, 2019, pp. 104-113.

[8].P. Senthilkumar, G. Yaswant, S. Kavitha, E. Chandramohan, G. Kowsalya, R. Vijay, B. Sudhagar, D. S. R. S. Kumar, Preparation and characterization of hybrid chitosansilver nanoparticles (Chi-Ag NPs); A potential antibacterial agent, International Journal of Biological Macromolecules, Vol. 141, 2019, pp. 290-297.

[9].R. G. Saratale, I. Karuppusamy, G. D. Saratale, A. Pugazhendhi, G. Kumar, Y Park, G. S. Ghodake, R. N. Bharagava, J. R. Banu, H. S. Shin, A comprehensive review on green nanomaterials using biological systems: Recent perception and their future applications, Colloids and Surfaces B: Biointerfaces, Vol. 170, 2018, pp. 20-35.

[10].R. Chen, Y. Li, M. Yan, X. Sun, H. Han, J. Li, J. Wang, L. Liu, K. Takahashi, Synthesis of hybrid zinc/silyl acrylate copolymers and their surface properties in the microfouling stage, RSC Advances, Vol. 6, 2016, pp. 13858-13866.

[11].P. Das, K. Ghosal, N. K. Jana, A. Mukherjee, P. Basak, Green synthesis and characterization of silver nanoparticles using belladonna mother tincture and its efficacy as a potential antibacterial and antiinflammatory agent, Materials Chemistry and Physics, Vol. 228, 2019, pp. 310-317.

[12].B. Kumar, K. Smita, L. Cumbal , A. Debut, Green synthesis of silver nanoparticles using Andean blackberry fruit extract, Saudi J Biol Sci, Vol. 24, 2017, pp. 45-50.

[13].R. Mythili, T. Selvankumar, S. KamalaKannan, C. Sudhakar, F. Ameen, A. AlSabri, K. Selvam, M. Govarthanan, H. Kim, Utilization of market vegetable waste for silver nanoparticle synthesis and its antibacterial activity, Materials Letters, Vol. 225, 2018, pp. 101-104. 
Lakhan M.N (et al.), Illicium verum as a green source for synthesis of silver nanoparticles and investigation of their antidiatom activity against Paeodactylum Tricornutum diatom

[14].K. Rajathi and A. Rajendran, Antimicrobial activity of silver nanoparticles from substituted and unsubstituted imidazolium ionic liquids, International Journal of Pharmaceutical Sciences Research, Vol. 8, 2017, pp. 4335-4340.

[15].F. A. Sheikha, M. A. Kanjwal, H. Kim, D. R. Pandeya, S. T. Hong, H. Y. Kim, Fabrication of titanium oxide nanofibers containing silver nanoparticles, Journal of Ceramic Processing Research, Vol. 11, 2010, pp. 685-691.

[16].A. H. Shar, M. N. Lakhan, J.Wang, M.Ahmed, K.T. Alali, R. Ahmed, I. Ali, A. Q. Dayo, Facile synthesis and characterization of selenium nanoparticles by the hydrothermal approach, Digest Journal of Nanomaterials and Biostructures, Vol. 14, 2019, pp. 867-872.

[17].B. Mahitha, B. D. P. Raju, G. R. Dillip, C. M. Reddy, K. Mallikarjuna, L. Manoj, S.
Priyanka, K. J. Raon, J. Sushma, Biosynthesis, characterization and antimicrobial studies of AgNPs extract from Bacopa monniera whole plant, Digest Journal of Nanomaterials and Biostructures, Vol. 6, 2011, pp. 135- 142.

[18].S. Zoha, M. Ahmad, S. J. A. Zaidi, M. N. Ashiq, W. Ahmad, T. J. Park, M. A. Basit, ZnO-based mutable $\mathrm{Ag} 2 \mathrm{~S} / \mathrm{Ag} 2 \mathrm{O}$ multilayered architectures for organic dye degradation and inhibition of E. coli and B. subtilis, Journal of Photochemistry \& Photobiology, A: Chemistry, 2020, pp. 1-27.

[19].K. Chand, M. I. Abro, U. Aftab, A. H. Shah, M. N. Lakhan, D. Cao, G. Mehdi, A. M. Ali Mohamed, Green synthesis characterization and antimicrobial activity against Staphylococcus aureus of silver nanoparticles using extracts of neem, onion and tomato, RSC Advances, Vol. 9, 2019 , pp. 17002-17015 